\title{
Patient choice, health policy or both?
}

\author{
Evidence for Health: From Patient Choice \\ to Global Policy \\ Anne Andermann \\ Cambridge University Press; 2013
}

66T T aving the right evidence at the right time in the
right format is critical to incorporating evidence into decisionmaking." We have all been exasperated by having inadequate time to synthesize information into a format that permits us to do our job, whether working as a physician looking for the best treatment for a patient, a decisionmaker modifying policy on the use of screening mammography or a funder reviewing program proposals to address undernutrition in children. Anne Andermann's book Evidence for Health: From Patient Choice to Global Policy is set in reality, yet it attempts the mammoth task of linking the concept of health decision-making in individual patient care with the complex process of balancing varying points of view - whether it be patients, or stakeholders from small institutions, larger jurisdictions or global health conundrums. Using clinical stories and policy dilemmas, Andermann illustrates how the rising number of stakeholders necessitates more trade-offs and consensus.

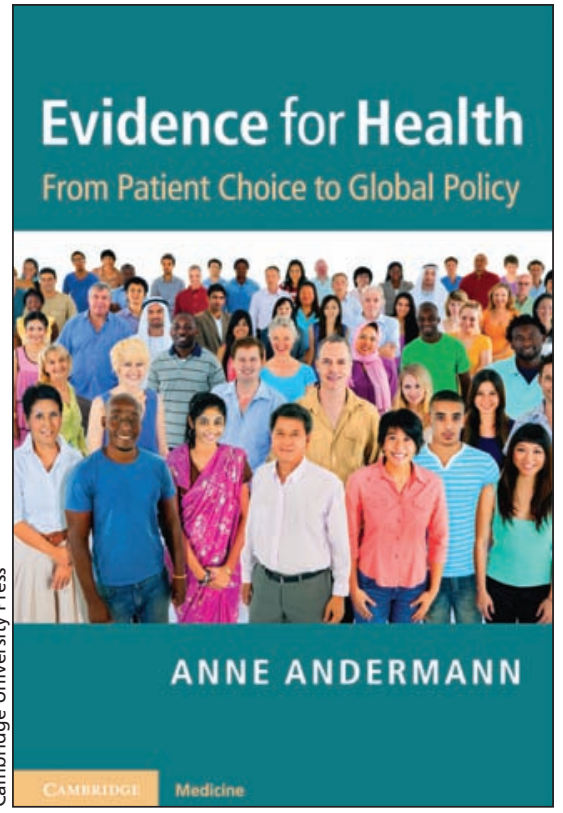

white, the stuff we know with certainty, like smoking cigarettes causes cancer. She then showcases the muddle of grey, the uncertainty in clinical and public health practice (e.g., evidence that no longer supports a protective effect of postmenopausal hormone use on cardiovascular health). How and when do we best integrate these observations?

The book begins by defining and addressing fundamental concepts such as individual and population health, determinants of health and the potential actions that mitigate these factors.

\section{How does one integrate evidence into health decisions when that evidence represents only part of an evolving story?}

This practical guide to evidenceinformed decision-making helps readers by clarifying and defining the notion of evidence, the creation of it, and its use for improving health. How does one integrate today's evidence into health decisions when that evidence represents only part of an evolving story at a particular point in time? Andermann describes the black and
Chapter 6 contains an eloquent portrayal of proximal risks versus upstream "causes" versus even-furtherupstream "causes," and how we might use these to assess a health problem like undernutrition in children. She also highlights the risk factors that would be targeted in global health if we were to consider risks associated with mortality versus daily adjusted life-years. If we were to consider the former, we would target hypertension, smoking and high blood glucose levels. If we were to consider the latter, we would emphasize underweight children, unsafe sex and alcohol use. Using detailed and thoughtfully organized tables and algorithms, Andermann delves into the types of evidence that can be acquired using complementary study designs and the health questions answered by these studies. A helpful yet short review of epidemiologic computations such as odds ratios, relative risks and number needed to treat follows. Andermann describes the inherent value and pitfalls of well-designed research and expounds that each type of study, if done well, contributes to the puzzle that ultimately represents the body of evidence used in decisionmaking. She highlights the factors that lead us to use evidence, and the barriers that inhibit us from adopting it. For example, predigested material from point-of-care tools enables us to consume the mountain of growing evidence. She also provides numerous links to evidence-based checklists for front-line health workers.

This book is unique because it is written for front-line health workers, policy-makers, global health decisionmakers and researchers. The author is a policy-maker and researcher who cares deeply about individual patient care.

Excellence requires that we all use evidence effectively. This book has the potential to help us use it better and to understand how others use it in their lines of work. For those who are not completely sated by this book, Andermann provides numerous excellent sources for the interested reader.

\section{Maureen Mayhew MD MPH}

Public health and clinical physician

Clinical Preventive Services

British Columbia Communicable

Disease Control

Vancouver, BC

CMAJ 2014. DOI:10.1503/cmaj.131628 